\title{
Hot molecular cores
}

\author{
Riccardo Cesaroni \\ INAF - Osservatorio Astrofisico di Arcetri, Largo Fermi 5, I-50125 Firenze, Italy \\ email: cesa@arcetri.astro.it
}

\begin{abstract}
The scope of this contribution is to review and discuss recent findings concerning the molecular environment of high-mass star forming regions. Special attention is devoted to "hot molecular cores" and their role in the formation of massive stars. After analysing the relationship between such cores and the surrounding molecular clumps, we discuss the results of interferometric observations of these objects and propose an evolutionary sequence proceeding from cold, pre-stellar cores to ultracompact Hiı regions.
\end{abstract}

Keywords. Stars: formation, ISM: clouds, ISM: molecules, ISM: jets and outflows

\section{Introduction}

It is commonly believed that a tight relationship exists between young massive (proto)stars and the molecular condensations named "hot molecular cores" (HMCs). These are usually observed in high-energy rotational lines of "rare" molecular species, probably evaporated from grain mantles by the strong radiation of deeply embedded early-type stars. Although HMCs were first detected at centimeter and millimeter wavelengths, in recent years an ever growing effort has been made to image these objects in the near- and mid-IR, where information on the embedded stellar component may be obtained. Beside observations, also theoretical studies have begun to take the HMC phenomenon into consideration as a fundamental "ingredient" in the process leading to the formation of high-mass stars (De Buizer et al. 2003; Kaufman et al. 1998; Linz et al. 2005; Pascucci et al. 2004; McKee \& Tan 2003). Notwithstanding the interest in the topic of HMCs, some confusion still exists on their nature and the role played by them in the context of massive star formation, where they are commonly believed to represent the precursors of ultracompact (UC) HII regions.

In an attempt of putting into context the various pieces of information on HMCs, in the following we will summarise the current knowledge on HMCs and discuss their properties. Special attention will be payed to the structure and physics of the cores, also in relation to the surrounding environment. The observational findings will be used to outline a possible scenario for the formation of early-type stars inside HMCs and eventually draw a tentative evolutionary scheme from pre-stellar cold cores to ultracompact (UC) HII regions.

\section{The environment of hot molecular cores}

Before proceeding with the present discussion, it is necessary to provide a definition of HMC. As a matter of fact, the identification of these objects is made on an observational basis, so that any definition must rely upon measurable quantities. Past experience has demonstrated that HMCs are powerful emitters in "exotic" molecules (see e.g. Kurtz et al. 2000), so that it seems reasonable to use these tracers as a distinctive feature of HMCs. Such molecules are usually detected in high-excitation transitions, which witness an origin from hot, dense gas. For the purpose of establishing the physical properties 
of the gas, $\mathrm{CH}_{3} \mathrm{CN}$ is certainly best suited, as it turns out to be an excellent density and temperature probe: being a symmetric-top molecule the ratio between different $K$ components provides us with an estimate of the temperature and optical depth, which makes it possible to identify HMCs even with single-dish observations (see e.g. the surveys by Olmi et al. 1993 and Araya et al. 2005). Interferometric follow-ups with arcsecond resolution have then be used to confirm the identifications (Olmi et al. 1996; Watt et al. 1999).

The exclusive association of HMCs with high-mass star forming regions, has been recently challenged by the discovery of similar objects, named "hot corinos" in a couple of low-mass protostars (Cazaux et al. 2003; Bottinelli et al. 2004). Although in all likelihood very similar to HMCs, hot corinos are $\sim 10^{6}$ times less massive and hence impossible to detect at distances typical of high-mass star forming regions, so that they will be ignored in the present contribution.

Kurtz et al. (2000) have selected from the literature 19 HMCs (see their Table 1), which will be used in the following as the reference list for this contribution, with the addition of a couple of objects, detected in recent studies. In summary, the main characteristics of HMCs, beside temperature $(\gtrsim 100 \mathrm{~K})$ and size $(\lesssim 0.1 \mathrm{pc})$, are their large masses $(\sim 10$ $\left.10^{3} M_{\odot}\right)$ and luminosities $\left(>10^{4} L_{\odot}\right)$, indicating that high-mass young stellar objects (YSOs) are significantly contributing to their (proto)stellar content. This conclusion is reinforced by the fact that $\mathrm{HMCs}$ are in all cases associated with $\mathrm{H}_{2} \mathrm{O}$ masers and $~ 50 \%$ of them also with one or more UC His regions.

Notwithstanding their clear identification, it is questionable that HMCs may be seen as "separate" physical entities. It seems instead that they correspond to density and temperature peaks inside more extended molecular clumps. The word "clump" will be used in the following to indicate the molecular surroundings of HMCs, the latter being located roughly at the center of the clumps. These have been observed in medium density tracers such as $\mathrm{C}^{34} \mathrm{~S}$ or $\mathrm{C}^{17} \mathrm{O}$ (Cesaroni et al. 1991; Plume et al. 1997; Hofner et al. 2000), are typically 10 times larger and more massive than HMCs, and have temperatures of the order of a few $10 \mathrm{~K}$. An example is shown is Fig. 1, where a $\mathrm{CH}_{3} \mathrm{CN}(5-4)$ image of the HMC in IRAS 20126+4104 is compared to a map of the surrounding clump, obtained in the $\mathrm{C}^{17} \mathrm{O}(2-1)$ line. Multi-line and continuum observations of the clumps have shown that the density and temperature vary as $n \propto R^{-p}$ and $T \propto R^{-q}$, with $R$ distance from the center (i.e. from the HMC) and $p \simeq 1.5-2.5, q \simeq 0.5$ (Hatchell et al. 2000; Fontani et al. 2002). While these laws are bound to break down on a sufficiently small scale, there is no indication that such a break occurs just at the surface of HMCs. Instead, Fontani et al. (2002) (see their Fig. 10) have shown that the mean HMC density is consistent with the density profile observed in the associated clumps. Furthermore, HMC velocities are not significantly different from those of the corresponding clumps so that HMCs cannot be seen as individual entities, free-floating inside the clumps.

If this is the case, what sets the diameters of HMCs? Since HMCs are traced by strong emission in high-excitation line of rare molecular species evaporated from the dust grains, the HMC diameter is related to the temperature of the dust, heated by the luminous stars inside the HMCs. One may estimate the size of the region inside which grain mantles are evaporated, assuming that this corresponds to a temperature $\geqslant 100 \mathrm{~K}$. The radius of such a region can be computed e.g. from Eq. (1) of Beckwith et al. (1990) and turns out to be e.g. $0.15 \mathrm{pc}$ for a O9 star and a dust emissivity index of 1 , in agreement with the typical size of HMCs. Similar values result also from the detailed modelling of the temperature structure of HMCs presented by Kaufman et al. (1998). One may conclude that HMCs are regions "enlightened" by the newly born high-mass stars embedded in the cores themselves. 


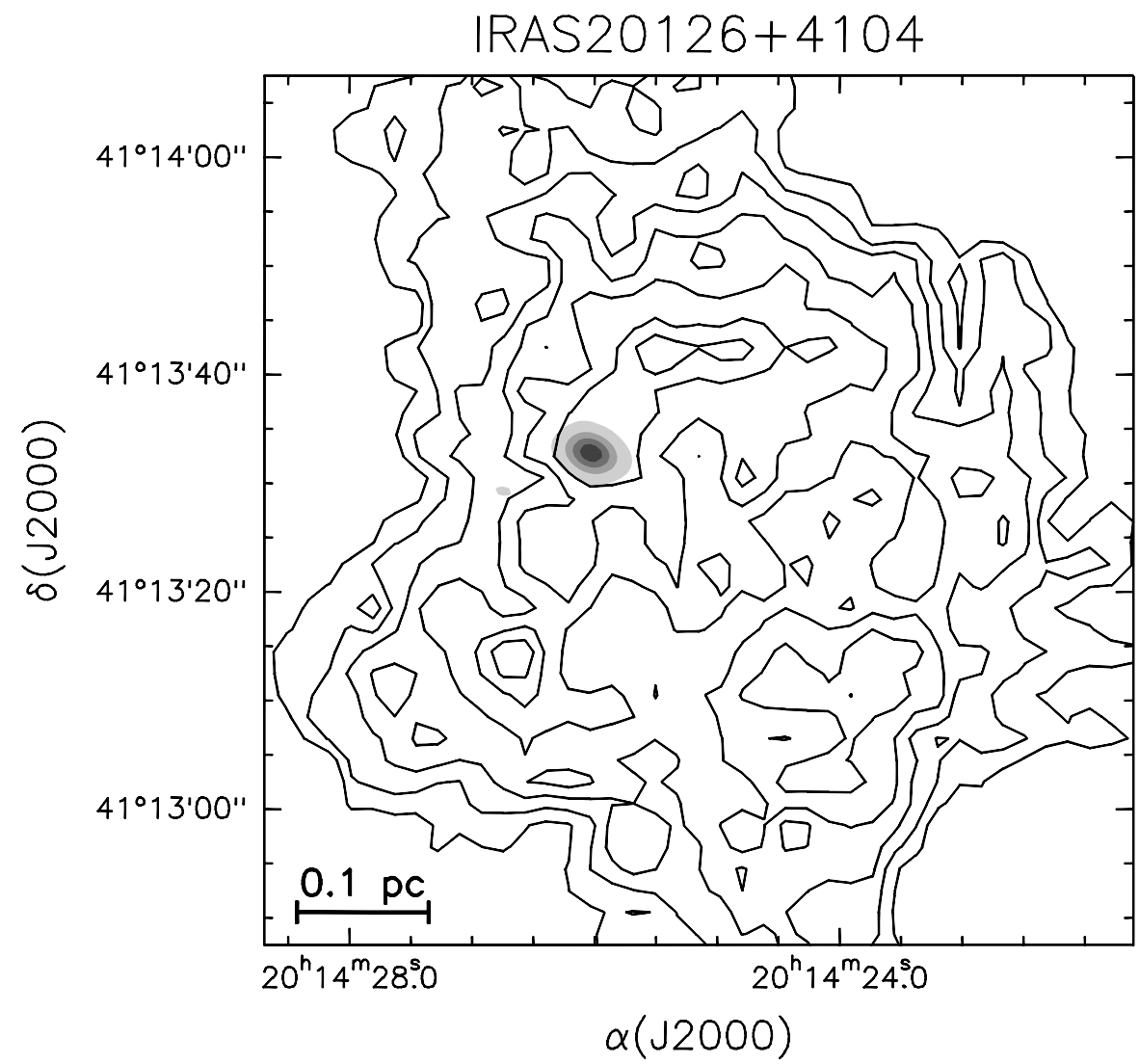

Figure 1. Maps of the HMC (grey scale) and surrounding molecular clump (contours) in the high-mass star forming region IRAS 20126+4104. The HMC and associated clump are traced respectively by the $\mathrm{CH}_{3} \mathrm{CN}(5-4)$ (Cesaroni et al. 1997) and $\mathrm{C}^{17} \mathrm{O}(2-1)$ (Fontani pers. comm.) lines.

If HMCs correspond to density and temperature enhancements at the center of parsecscale molecular clumps, their formation and evolution strictly depend on the physical status of the clump. Recently, Fontani et al. (2002) have performed a detailed comparison between the mass of a number of clumps and the corresponding virial estimates (see their Fig. 6): in all cases the latter are significantly larger than the former, indicating that the clumps are marginally unstable. The combination of this result with the steep density profiles, resembles the situation of the singular isothermal sphere depicted by Shu et al. (1987) and suggests that the clumps might be undergoing inside-out collapse. In this scenario HMCs would mark the center of collapse, where presumably fragmentation and star formation is going on. This is not too different from the scenario hypothesised by McKee \& Tan (2003), where massive star formation occurs in small cores of a few $10 M_{\odot}$, located inside large scale molecular clumps similar to those previously described.

\section{The stellar content of hot molecular cores}

In order to elaborate further on HMCs as sites of (massive) star formation, it is worth analysing the relationship between the cores and their stellar content. This can be done by comparing the luminosity to the mass of HMCs, because the former originates from the embedded stars, whereas the latter corresponds to the mass of the gas, calculated 


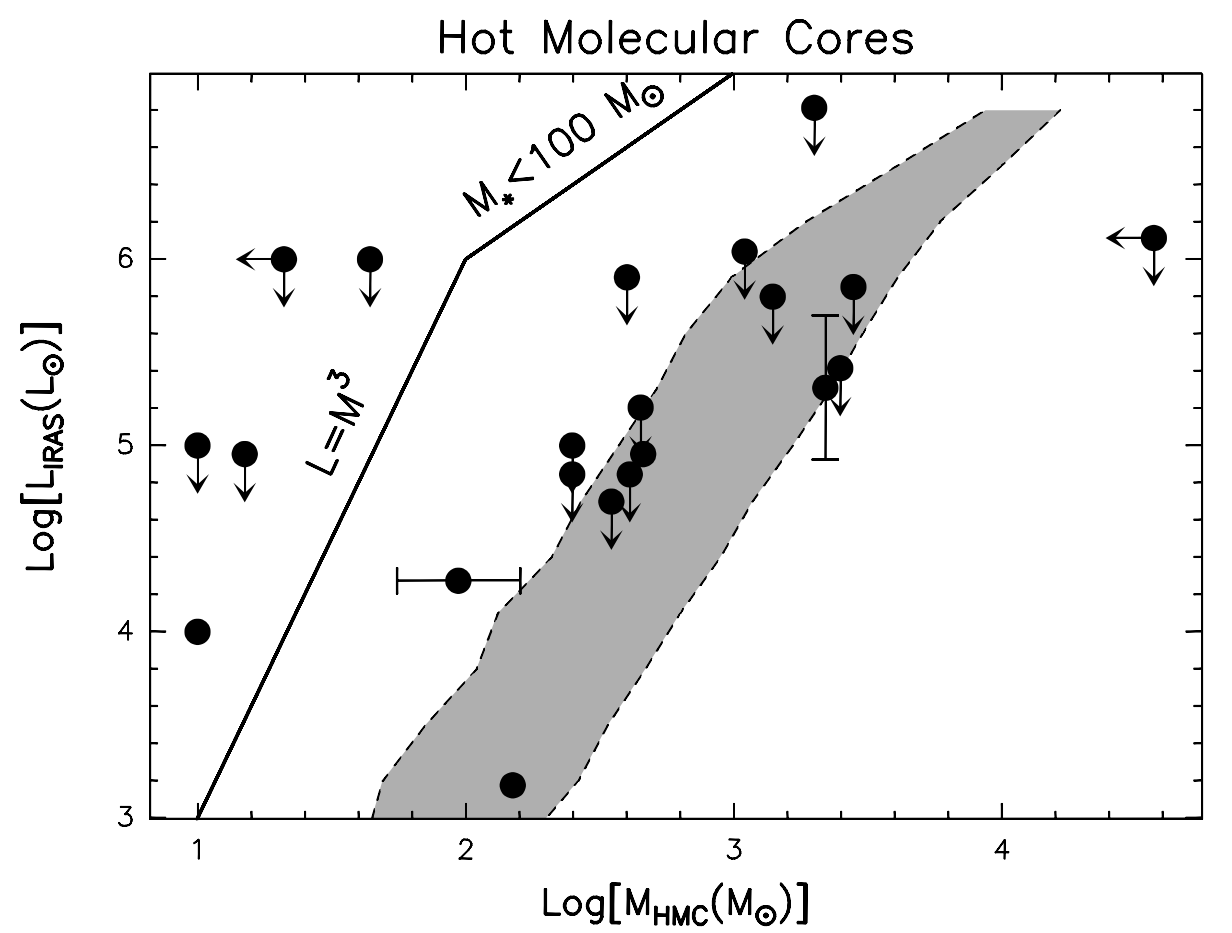

Figure 2. Plot of luminosity versus mass for a sample of HMCs. The solid lines mark the luminosity attainable by concentrating the whole mass of the HMC into a minimum number of stars having mass $\leqslant 100 M_{\odot}$. The shaded area denotes the luminosity of a stellar cluster with total mass equal to that of the HMC and distributed according to the IMF of Scalo (1998), with a minimum stellar mass of $0.3 M_{\odot}$.

from the millimeter continuum emission. Figure 2 is an updated version of Fig. 3 of Kurtz et al. (2000), displaying a plot of the luminosity versus mass for our sample of bona fide HMCs (see Sect.2). Note that in the large majority of cases the luminosity is obtained from the IRAS measurements and hence is to be taken as an upper limit, due to the limited angular resolution. The straight lines mark the luminosity expected assuming that the whole HMC mass goes into stars $\leqslant 100 M_{\odot}$, while the shaded area indicates the region occupied by clusters of zero-age main-sequence stars, assuming a Scalo (1998) initial mass function (IMF). Clearly, two groups of HMCs are seen: those lying above the solid lines and those occupying the shaded area. Noticeably, the former are "light" HMCs, below a few $\sim 10 M_{\odot}$, whereas the latter are all "heavy", i.e. above $100 M_{\odot}$. This distinction is a clue to explain the different behaviour of the HMCs in the plot. Let us consider in turn "light" and "heavy" HMCs.

"Light" HMCs lie in a forbidden region: in fact, no zero-age main-sequence star with mass below $100 M_{\odot}$ may exceed the luminosity indicated by the solid lines in the figure. A plausible explanation is that the stellar mass is underestimated. Indeed, the mass used in the plot is not the stellar mass, but rather the mass of the gas, obtained from measurements of the millimeter continuum emission. This implies that the mass of the star is comparable to or greater than the gas mass in the HMC. We conclude that "light" HMCs are associated with one (or few) massive stars.

The distribution of the "heavy" HMCs in Fig. 2 suggests that their luminosities originate from a cluster of stars, whose total mass is equal to the mass of the HMC. In fact, if the mass of the stars were only, e.g., a factor 3 less than that of the core, the points in 
the figure would move to the left, very close to the forbidden region marked by the solid lines. The conclusion is that in the "heavy" HMCs the mass of the stars is comparable to the mass of the gas. Consequently, the star formation efficiency must be very high and proceed in clustered mode.

Is there any direct observational evidence for the previous conclusions? Given the distance to high-mass star forming regions (typically a few $\mathrm{kpc}$ ) sub-arcsecond resolution is necessary to resolve the structure of HMCs and possibly identify deeply embedded YSOs. Such a result is difficult to achieve with the interferometers available to date, and certainly very time consuming. That explains why only a handful of sources have been deeply investigated so far, of which the Orion HMC is certainly the best studied (see e.g. Beuther et al. 2004 and references therein) being by far less distant than the others. In this case, the most massive star identified so far corresponds to radio source I, whose mass is $\leqslant 10 M_{\odot}$, comparable to that of the HMC $\left(\sim 10 M_{\odot}\right)$, as expected for "light" HMCs. Another interesting object is IRAS 20126+4104, a "light" HMC associated with a powerful bipolar outflow/jet (Cesaroni et al. 1997; Zhang et al. 1998b; Cesaroni et al. 1999; Hofner et al. 1999; Shepherd et al. 2000; Moscadelli et al. 2005). The core has been found to be undergoing Keplerian rotation about the jet/outflow axis (Cesaroni et al. 2005): this fact not only allows one to derive the (proto)stellar mass $\left(7 \pm 3 M_{\odot}\right)$, but also to establish that in all likelihood only one massive star (or a binary system) is present in the innermost 2000 au of the HMC.

Two "heavy" HMCs (G10.47+0.03 and G31.41 + 0.31) have been recently imaged with sub-arcsecond resolution in the $\mathrm{NH}_{3}(4,4)$ and $\mathrm{CH}_{3} \mathrm{CN}(12-11)$ lines and in the centimeter and millimeter continuum (Cesaroni et al. 1998; Beltrán et al. 2004; Beltrán et al. 2005; Hofner et al. in prep.; Beltrán et al. in prep.). The results indicate the existence of multiple, deeply embedded UC and hypercompact HiI regions in both cores, supporting the idea that a cluster of massive stars is forming in these HMCs. Both objects seem to be associated with molecular outflows, and G31.41+0.31 presents also a remarkable velocity gradient interpreted as rotation by Beltrán et al. (2005) (but see Gibb et al. 2004 for a different interpretation). If this is the case, one may speculate that infall plus conservation of angular momentum have induced flattening and rotation of the core, thus creating an accreting toroid around the newly born (proto)stars.

\section{A tentative scenario for massive star formation}

We now attempt to elaborate on the previous findings and propose a scenario for the formation of massive stars taking into account the characteristics of clumps and HMCs. These may be summarised as follows:

Clumps: diameter $\sim 1$ pc; mass $\sim 10^{3} M_{\odot}$; mean density $10^{5} \mathrm{~cm}^{-3}$, with steep density profile, roughly $n \propto R^{-2}$; typical densities $\sim 10^{5} \mathrm{~cm}^{-3}$, implying a free-fall time of $\sim 10^{5} \mathrm{yr}$; temperatures $\sim 30 \mathrm{~K} ; M_{\text {gas }}>M_{\text {virial }}$, implying instability of the clumps unless a strong (a few $\mathrm{mG}$ ) magnetic field is present.

HMCs: located close to the center of the clumps; 10 times smaller and less massive, and 100 times denser than the corresponding clumps, consistently with $n \propto R^{-2}$; typical temperatures $\sim 100 \mathrm{~K}$; evidence for rotation with $M_{\mathrm{HMC}}>M_{\mathrm{dyn}}$, where $M_{\mathrm{dyn}}$ is the mass required for balancing centrifugal and gravitational forces; multiple embedded stars with $M_{\text {stars }} \simeq M_{\text {gas }}$.

Although other explanations are possible, it is tempting to interpret the the density profile of the clump as a sign of inside-out collapse, as depicted by Shu et al. (1987). Naïvely, one may imagine that the HMC is located inside the infalling region: it is in fact interesting to note that in the HMC the stellar mass equals the gas mass, as well as in 
the singular isothermal sphere the infalling mass is about the same as the mass of the accreting protostar.

Of course the situation is much more complex in the case of clumps and HMCs than for the singular isothermal sphere, as in the latter only one star is formed, whereas in the former the gas seems to fragment on a small scale and give rise to a group of massive stars.

With this in mind, one may speculate that the formation of massive stars occurs according to the following scenario:

- an unstable clump undergoes inside-out collapse on a free-fall time $\sim 10^{5} \mathrm{yr}$;

- the border of the infalling region expands at a speed of a few $\mathrm{km} \mathrm{s}^{-1}$;

- multiple star formation (low- and high-mass) occurs inside the infalling region, with accretion rates of order $M_{\text {clump }} / t_{\text {free-fall }} \simeq 10^{-2} M_{\odot} \mathrm{yr}^{-1}$;

- conservation of angular momentum speeds up the rotation of the HMC and favours fragmentation on a scale equal to the centrifugal radius (see Terebey et al. 1984)

$$
R_{\mathrm{c}}(\mathrm{pc})=232.6 v_{\mathrm{rot}}^{2}\left(\mathrm{~km} \mathrm{~s}^{-1}\right) R_{\mathrm{HMC}}^{2}(\mathrm{pc}) / M_{\mathrm{HMC}}\left(M_{\odot}\right) \simeq 0.2 R_{\mathrm{HMC}}(\mathrm{pc}) ;
$$

- if the steep density profile is preserved in the HMC, the mass inside a centrifugal radius scales linearly with the radius itself and is hence $\sim 5$ times smaller than the HMC mass, i.e. $\sim 20 M_{\odot}$;

- the number of fragments (and hence of stars of all masses, assuming $100 \%$ star formation efficiency) is hence $\sim 100$;

- the accretion rate per star is $10^{-4} M_{\odot} \mathrm{yr}^{-1}$, sufficient to form a $10 M_{\odot}$ star in $10^{5} \mathrm{yr}$.

In conclusion, while initially the formation of early-type stars in a clump may proceed through the formation of one or a few "heavy" HMCs, eventually these will fragment into a large number of sub-cores with sizes comparable to the centrifugal radius of the HMC. The sub-cores will have a mass spectrum similar to the stellar IMF (analogous to what found in low-mass star forming regions by Testi \& Sargent 1998 and Motte et al. 1998 and in a high-mass object by Beuther \& Schilke 2004) and the most massive of these will undergo Keplerian rotation around an early-type star. It is worth noting that the centrifugal radius and associated mass estimated above compare well to the radius and mass of the disk associated with the massive protostar IRAS 20126+4104 (Cesaroni et al. 2005).

The tentative scenario depicted above raises several questions. First of all, application of the singular isothermal sphere seems inappropriate in this case, given the existence of a temperature gradient in the clumps: approximately $T \propto R^{-0.5}$. However, this mirrors the present situation, after the onset of star formation. Before that, the temperature was very likely constant, as suggested by the recently detected IR-dark clouds (Egan et al.1998; Garay et al.2004) which may represent the precursors of the molecular clumps discussed in the present study. The timescale for heating up the clump is much shorter than the dynamical time scale $\left(\sim 10^{5} \mathrm{yr}\right)$, which proves that the density profile cannot change significantly over a clump's life time.

Another problem is the lack of iron-clad evidence for infall in the clumps. Infall should proceed at a free-fall velocity of several $\mathrm{km} \mathrm{s}^{-1}$, which should be clearly seen as redshifted self-absorption in the profiles of lines as broad as a few $\mathrm{km} \mathrm{s}^{-1}$. However, inward motions may exist only inside the border of the infalling region, which is limited to the inner portion of the clump. Most of this is probably filled by the $\mathrm{HMC}(\mathrm{s})$. Indeed, some evidence for infall inside HMCs has been reported (see e.g. Keto et al. 2002, Zhang et al. 1998a, Cesaroni et al. 1999, Beltrán et al. 2005, and Cesaroni et al. 2005). It must be 
also stressed that the detection of infall is complicated by the presence of outflow, on a large scale, and rotation, on a small scale.

Finally, what determines the end of the accretion phase on HMCs? In other words, what sets a limit of a few $100 M_{\odot}$ on the mass of a HMC? In fact, if accretion proceeded for $10^{5} \mathrm{yr}$, eventually all of the clump's mass $\left(\gtrsim 10^{3} M_{\odot}\right)$ would collapse into the HMC. The end of the HMC phase is probably determined by the onset of powerful molecular outflows and the subsequent development of UC HII regions, whose estimated expansion time $\left(10^{5} \mathrm{yr}\right.$; Wood \& Churchwell 1989) is comparable to the clump life time.

\section{G24.78+0.08: a benchmark for high-mass star formation}

\subsection{Confronting the star formation scenario with observations}

Is there any direct observational evidence in favour of the speculative scenario proposed above? The case of the high-mass star forming region G24.78+0.08 may be used for this purpose.

This region is located at a distance of $7.7 \mathrm{kpc}$, has a large bolometric luminosity $\left(\lesssim 7 \times 10^{4} L_{\odot}\right)$, and is characterised by the presence of two closeby UC HII regions. A number of maser spots have been also detected from various molecular species. In particular, $\mathrm{H}_{2} \mathrm{O}$ masers are gathered in two groups, one close to the most compact UC HII region, the other offset from it by $\sim 0.34 \mathrm{pc}$. All of this proves the existence of an embedded population of high-mass YSOs spread over $\sim 0.4 \mathrm{pc}$ and have triggered an accurate study of the region through a large number of low- and high-angular resolution observations (Codella et al. 1997; Furuya et al. 2002; Cesaroni et al. 2003; Beltrán et al. 2004; Beltrán et al. 2005).

This has made it possible to analyse the properties of the molecular gas from $1 \mathrm{pc}$ to $0.03 \mathrm{pc}$, thus providing an excellent test for the scenario proposed in Sect. 4. As expected, on a large scale the massive YSOs are surrounded by a medium density $\left(\sim 10^{6} \mathrm{~cm}^{-3}\right)$ clump with diameter $\sim 1 \mathrm{pc}$ and mass $\sim 4000 M_{\odot}$, significantly larger than the corresponding virial mass of $2900 M_{\odot}$. The volume density varies as $n \propto R^{-1.8}$, consistently with a singular isothermal sphere undergoing inside-out collapse.

In the innermost region (i.e. for $R<0.15 \mathrm{pc}$ ), the approximate spherical symmetry of the clump breaks up into a number of compact cores, having a total mass $M_{\text {cores }} \simeq 900 M_{\odot}$. This suggests that such a region could coincide with that undergoing infall. Assuming that the gas has collapsed into the cores, the mass accretion rate $\left(\dot{M}_{\mathrm{accr}} \simeq 3 \times 10^{-2} M_{\odot} \mathrm{yr}^{-1}\right)$ is given by Eq. (23) of Shu et al. (1987) for a line width of $\sim 5 \mathrm{~km} \mathrm{~s}^{-1}$, and the border of the infalling region $\left(R_{\text {inf }}\right)$ expands at a speed $\left(v_{\exp } \simeq 5 \mathrm{~km} \mathrm{~s}^{-1}\right)$ equal to the line width,

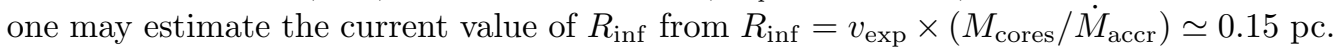
This compares well with the region over which the cores are distributed, thus leading support to the infall scenario.

As discussed in Sect. 4, massive star formation could proceed through core fragmentation at a level of the centrifugal radius of each core. Is there any evidence for infall, rotation, and star formation in the cores detected in G24.78+0.08?

As already mentioned, two UC HII regions and four cores have been identified by means of high-angular resolution observations of $\mathrm{CS}$ and $\mathrm{CH}_{3} \mathrm{CN}$ and of the continuum emission at centimeter and millimeter wavelengths (Furuya et al. 2002; Beltrán et al. 2004). Two of the cores (G24A1 \& G24A2) present prominent emission in the $\mathrm{CH}_{3} \mathrm{CN}$ lines and turn out to have high temperatures $(\sim 120 \mathrm{~K})$, so that they may safely be classified as HMCs. One of this (G24A1) is associated with the strongest UC HiI region, while the other (G24A2) presents strong pointlike emission from 3.6 to $8 \mu \mathrm{m}$ (data retrieved from 
the GLIMPSE database). Both are associated with $\mathrm{H}_{2} \mathrm{O}$ and $\mathrm{CH}_{3} \mathrm{OH}$ masers and with a bipolar molecular outflow, although it cannot be established from which of the cores the latter originates. There is little doubt that G24A1 and G24A2 are two high-mass star forming HMCs.

It is clear that high-mass star formation is very active in the G24.78+0.08 cores and the presence of powerful outflows supports the existence of infall. The $\mathrm{CH}_{3} \mathrm{CN}$ line width in G24A1 is maximum at the center of the core: this may be an optical depth effect, but is also consistent with infall, as in this case the maximum velocity dispersion along the line of sight is attained just for the line of sight passing through the center.

Clear evidence for rotation has been found in G24A1 and G24A2. This is seen as a velocity gradient measured in the $\mathrm{CH}_{3} \mathrm{CN}$ lines, perpendicular to the direction of the corresponding outflow axes, as shown in Fig. 3. Numerical simulations have demonstrated that the rotation velocity varies most likely linearly with $R$, consistently with the fact that Keplerian rotation is impossible for disks more massive than $\sim 100 M_{\odot}$. The rotating cores are hence self-gravitating massive disks, which cannot be supported by centrifugal forces. In this situation, coherently with the scenario in Sect. 4, fragmentation on a centrifugal radius several times smaller than the core radius may occur.

With this in mind, it is interesting to note that recent EVN observations (Moscadelli pers. comm.) of the $\mathrm{CH}_{3} \mathrm{OH}$ masers in G24A1 and G24A2 have revealed that the maser spots are distributed consistently with the $\mathrm{CH}_{3} \mathrm{CN}$ velocity gradient, although over regions $\sim 3$ times smaller $(0.023$ and $0.020 \mathrm{pc})$ and covering velocity ranges $\sim 3$ times larger (9.1 and $\left.5.7 \mathrm{~km} \mathrm{~s}^{-1}\right)$. This is consistent with conservation of angular momentum from the large to the small scale inside the cores: indeed the centrifugal diameters $(0.030$ and $0.012 \mathrm{pc}$ ) and corresponding velocities (13 and $7.4 \mathrm{~km} \mathrm{~s}^{-1}$ ) of the G24A1 and G24A2 disks agree with those of the two groups of $\mathrm{CH}_{3} \mathrm{OH}$ maser spots. The dynamical masses needed for equilibrium are 55 and $19 M_{\odot}$ for G24A1 and G24A2 respectively. From the free-free continuum emission of the UC HII region in G24A1, Codella et al. (1997) estimate a spectral type $\mathrm{O} 9.5$ for the exciting star, corresponding to a stellar mass of $\sim 20 M_{\odot}$. This must be summed to the mass of the gas inside $0.023 \mathrm{pc}$, which is $31 M_{\odot}$ for a mean density of $10^{8} \mathrm{~cm}^{-3}$. The total mass (star plus gas) is consistent with the $55 M_{\odot}$ computed from the $\mathrm{CH}_{3} \mathrm{OH}$ velocity spread in G24A1.

\subsection{Possible evolutionary sequence}

Beside G24A1 and G24A2, also another core, G24C, has been found in the G24.78+0.08 region (see Fig. 3). Unlike the two HMCs, this has a relatively low temperature $(\sim 30 \mathrm{~K})$ and faint $\mathrm{CH}_{3} \mathrm{CN}$ emission. However, various facts indicate that also this core might be forming high-mass stars: its mass is comparable to that of the two HMCs $\left(\sim 200 M_{\odot}\right)$, is associated with $\mathrm{H}_{2} \mathrm{O}$ maser emission, and powers a bipolar molecular outflow with properties typical of outflows from massive stars. Also in this case, evidence for rotation about the outflow axis is seen, this time in the CS(3-2) line (Beltrán et al. 2004).

A fourth core, G24D, is detected only in the millimeter continuum, which does not allow a temperature estimate. Assuming a temperature $\leqslant 30 \mathrm{~K}$, the mass is $\geqslant 100 M_{\odot}$, suggestive of a cold, high-mass protostellar core.

Finally, it is worth noting that the UC HII region not associated with G24A1, named G24B by Codella et al. (1997), presents only free-free emission not only at centimeter wavelengths but also in the millimeter. This indicates that unlike the other UC HII region, G24B is not embedded in a dense, dusty core.

Given the relatively large number of objects revealed in the G24.78+0.08 clump, one may attempt a classification sorting G24A1-D according to a possible evolutionary sequence. In fact, in this case one is dealing with sources formed in the same environment, 


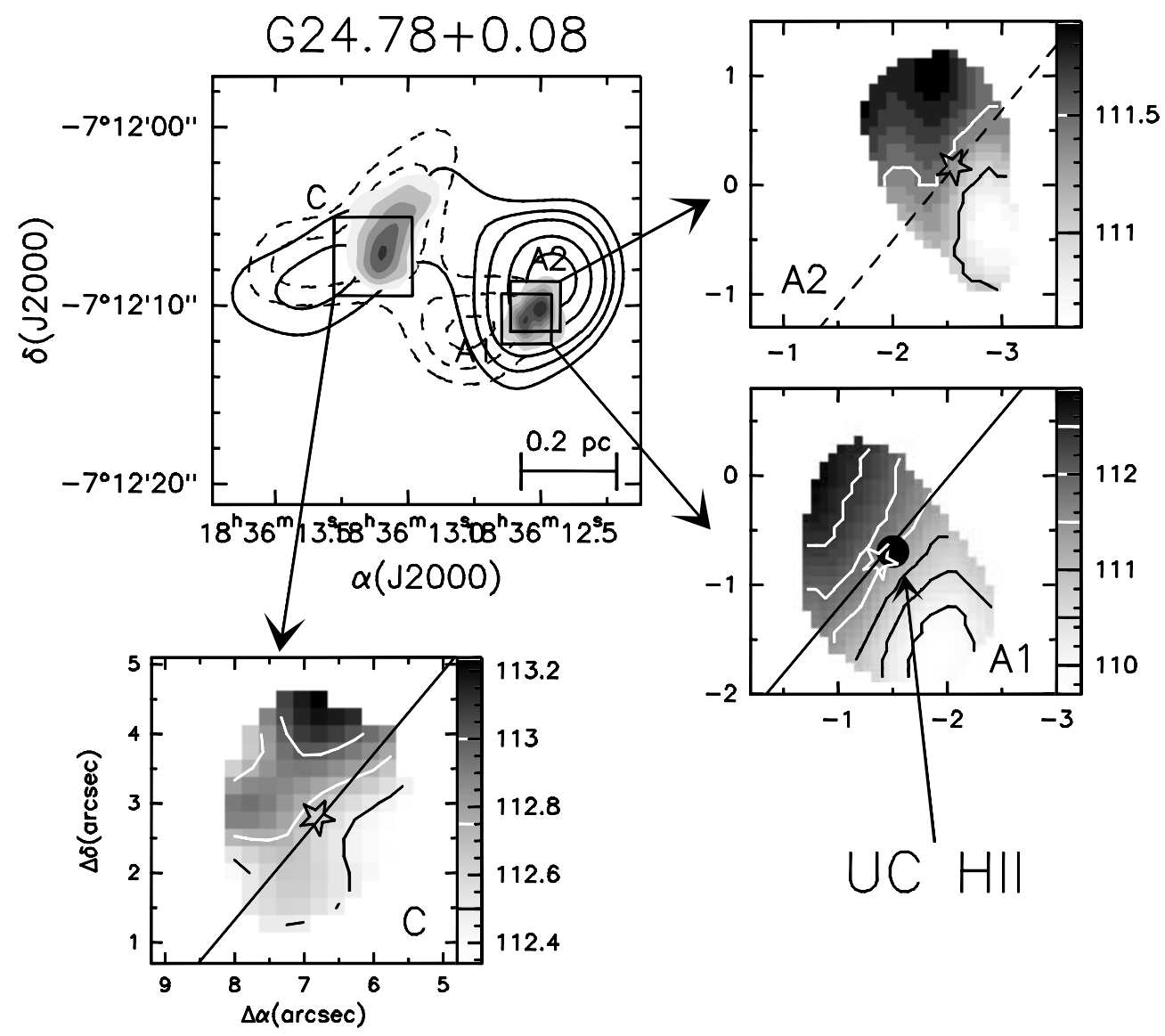

Figure 3. Disk/outflow systems in the high-mass star forming region G24.78+0.08 (Beltrán et al. 2004). The straight lines represent the axes of the bipolar outflows detected by Furuya et al. (2002). The starred symbols indicate the positions of the millimeter continuum peaks, while the filled circle in G24 A1 represents the UC Hiı region detected by Codella et al. (1997). Top left: Maps of the outflows (contours) overlayed on images of the CS(3-2) (towards G24C) and $\mathrm{CH}_{3} \mathrm{CN}(12-11)$ (towards G24A1 and G24A2) emission integrated under the line. Solid and dashed contours correspond respectively to blue- and red-shifted gas. Bottom left: Map of the CS(3-2) line velocity observed by Cesaroni et al. (2003) towards G24 C. The grey scale is expressed in $\mathrm{km} \mathrm{s}^{-1}$. Top and bottom right: Maps of the $\mathrm{CH}_{3} \mathrm{CN}(12-11)$ line velocity towards G24 A1 and G24 A2.

so that any difference between them may be attributed more likely to age effects than to different initial conditions. Table 1 summarises the properties of G24A1-D in order to outline similarities and differences. For each object we report temperature, presence of dust continuum and/or line emission, and association with outflows, disks, masers and UC HII regions. As the star formation process goes on, the temperature should increase as a consequence of the increasing stellar luminosities, outflows should appear in association with rotating accretion disks, and eventually UC HII regions should form and expand determining the destruction of the HMC.

These facts suggest the sequence outlined in Table 1: G24 D $\rightarrow \mathrm{C} \rightarrow \mathrm{A} 2 \rightarrow \mathrm{A} 1 \rightarrow$ B. G24D is presumably cold, being detected only in the millimeter continuum but not in molecular tracers; also, it is not associated with any signpost of star formation, so that we believe it to be the youngest. G24C is warm and associated with a disk/outflow, consistently with the presence of $\mathrm{H}_{2} \mathrm{O}$ (Forster \& Caswell 1989) and class $\mathrm{I} \mathrm{CH}_{3} \mathrm{OH}$ masers 
Table 1. Summary of properties of the cores in the G24.78+0.08 high-mass star forming region

\begin{tabular}{|c|c|c|c|c|c|}
\hline Object name & G24D & G24C & G24A2 & G24A1 & G24B \\
\hline temperature $(\mathrm{K})$ & - & 30 & 120 & 120 & - \\
\hline dust emission & $\mathbf{Y}$ & $\mathbf{Y}$ & $\mathbf{Y}$ & $\mathbf{Y}$ & $\mathrm{N}$ \\
\hline line emission & $\mathrm{N}$ & $\mathbf{Y}$ & $\mathbf{Y}$ & $\mathbf{Y}$ & $\mathrm{N}$ \\
\hline bipolar outflow & $\mathrm{N}$ & $\mathbf{Y}$ & $\mathbf{Y}^{a}$ & $\mathbf{Y}^{a}$ & $\mathrm{~N}$ \\
\hline rotating disk & $\mathrm{N}$ & $\mathbf{Y}$ & $\mathbf{Y}$ & $\mathbf{Y}$ & $\mathrm{N}$ \\
\hline $\mathrm{H}_{2} \mathrm{O}$ maser & $\mathrm{N}$ & $\mathbf{Y}$ & $\mathbf{Y}$ & $\mathbf{Y}$ & $\mathrm{N}$ \\
\hline class $\mathrm{I} \mathrm{CH}_{3} \mathrm{OH}$ maser & $\mathrm{N}$ & $\mathbf{Y}$ & $\mathbf{Y}^{a}$ & $\mathbf{Y}^{a}$ & $\mathrm{~N}$ \\
\hline class II $\mathrm{CH}_{3} \mathrm{OH}$ maser & $\mathrm{N}$ & $\mathrm{N}$ & $\mathbf{Y}$ & $\mathbf{Y}$ & $\mathrm{N}$ \\
\hline UC HiI region & $\mathrm{N}$ & $\mathrm{N}$ & $\mathrm{N}$ & $\mathbf{Y}$ & $\mathbf{Y}$ \\
\hline
\end{tabular}

${ }^{a}$ could be associated with either or both G24A1 and/or G24A2.

(Beltrán pers. comm.), which are also believed to be outflow tracers. Class II $\mathrm{CH}_{3} \mathrm{OH}$ maser emission is detected only in G24A1 and G24A2 (Walsh et al. 1998; Moscadelli pers.

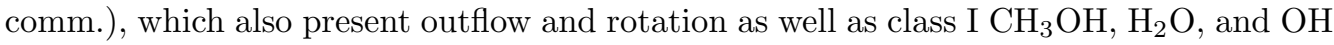
maser emission: the high temperature of these two cores suggests that luminous stars have already formed in them, as proved by the strong mid-IR source detected in G24A2. However, G24A1 is likely more evolved as witnessed by the presence of an embedded UC HII region. Finally, G24B is detected only in the free-free continuum, suggesting that this UC HII region is old enough to have dispersed the parental core.

The evolution from G24D to G24B must occur over a free-fall time of the parsec-scale clump, so that the age spread between these objects cannot exceed $\sim 10^{5} \mathrm{yr}$.

\section{Conclusions}

The present knowledge of HMCs suggests that these objects could highlight the very sites of massive star formation inside parsec-scale clumps undergoing collapse in their innermost regions. In fact, comparison between the masses and luminosities of HMCs indicates that their masses are comparable to those of the embedded stellar clusters. Although highly speculative at the moment, recent observations seem to lead support to a star formation scenario according to which HMCs would lie in the collapsing region of the clumps, where the infalling gas spins up for conservation of angular momentum. This process could eventually lead to fragmentation of the HMC over a scale characterised by equilibrium between gravitational and centrifugal forces. The fragments may then form accretion disks rotating about the newly formed stars. The increasing evidence of outflows, infall, and rotation in high-mass star forming regions on scales smaller than $0.1 \mathrm{pc}$ looks like a promising forward for more sensitive and higher resolution studies of HMCs with new generation instruments such as ALMA.

\section{Acknowledgements}

It is a pleasure to thank Leonardo Testi for calculating the mass-luminosity relationship for a stellar cluster shown in Fig. 2 and Malcolm Walmsley for stimulating discussions and a critical reading of the manuscript.

\section{References}

Araya, E., Hofner, P., Kurtz, S., Bronfman, L., DeDeo, S. 2005, ApJS 157, 279

Beckwith, S. V. W., Sargent, A. I., Chini, R. S., Guesten, R. 1990, AJ 99, 924 
Beltrán, M.T., Cesaroni, R., Neri, R., Codella, C., Furuya, R.S., Testi, L., Olmi, L. 2004, ApJ 601, L187

Beltrán, M.T., Cesaroni, R., Neri, R., Codella, C., Furuya, R.S., Testi, L., Olmi, L. 2005, A\&A in press

Beuther, H., Schilke, P. 2004, Science 303, 1167

Beuther, H., Zhang, Q., Greenhill, L.J., Reid, M.J., Wilner, D., Keto, E., Marrone, D., Ho, P.T.P., Moran, J.M., Rao, R., Shinnaga, H., Liu, S.-Y. 2004, ApJ 616, L31

Bottinelli, S., Ceccarelli, C., Lefloch, B., Williams, J.P., Castets, A., Caux, E., Cazaux, S., Maret, S., Parise, B., Tielens, A.G.G.M. 2004, ApJ 615, 354

Cazaux, S.; Tielens, A. G. G. M.; Ceccarelli, C.; Castets, A.; Wakelam, V.; Caux, E.; Parise, B.; Teyssier, D. 2003, ApJ 593, L51

Cesaroni, R., Walmsley, C.M., Kömpe, C., Churchwell, E. 1991, A\&̛A 252, 278

Cesaroni, R., Felli, M., Testi, L., Walmsley, C.M., Olmi, L. 1997, A $\mathscr{G} A$ 325, 725

Cesaroni, R., Hofner, P., Walmsley, C.M., Churchwell, E. 1998, A\&A 331, 709

Cesaroni, R., Felli, M., Jenness, T., Neri, R., Olmi, L., Robberto, M., Testi, L., Walmsley, C.M. 1999, A\&A 345, 949

Cesaroni, R., Codella, C., Furuya, R.S., Testi, L. 2003, A\& A 401, 227

Cesaroni, R., Neri, R., Olmi, L., Testi, L., Walmsley, C.M., Hofner, P. 2005, A\&̋A 434, 1039

Codella, C., Testi, L., Cesaroni, R. 1997, A\&SA 325, 282

De Buizer, J. M., Radomski, J. T., Telesco, C. M., Piña, R. K. 2003, ApJ 598, 1127

Fontani, F., Cesaroni, R., Caselli, P., Olmi, L. 2002, A\&SA 389, 603

Forster, J.R. \& Caswell, J.L. 1989, A\&A 213, 339

Furuya, R. S., Cesaroni, R., Codella, C., Testi, L., Bachiller, R., Tafalla, M. 2002, A\&SA 390, L1

Egan, M. P., Shipman, R. F., Price, S. D., Carey, S. J., Clark, F. O., Cohen, M. 1998, ApJ 494, L199

Garay, G., Faúndez, S., Mardones, D., Bronfman, L., Chini, R., Nyman, L. 2004, ApJ 610, 313

Gibb, A.G., Wyrowski, F., Mundy, L.G. 2004, ApJ 616, 301

Hatchell, J., Fuller, G. A., Millar, T. J., Thompson, M. A., Macdonald, G. H. 2002, A 6 A 357, 637

Hofner, P., Cesaroni, R., Rodríguez, L.F., Martí, J. 1999, A\&̋A 345, L43

Hofner, P., Wyrowski, F., Walmsley, C.M., Churchwell, E. 2000, ApJ 536, 393

Kaufman, Michael J., Hollenbach, David J., Tielens, A. G. G. M. 1998, ApJ 497, 276

Keto, E. 2002, ApJ 568, 754

Kurtz, S., Cesaroni, R., Churchwell, E., Hofner, P., Walmsley, M. 2000, Protostars and Planets IV p. 299

Linz, H., Stecklum, B., Henning, Th., Hofner, P., Brandl, B. 2005, A\&\&A 429, 903

McKee, C. F., Tan, J. C. 2003, ApJ 585, 850

Moscadelli, L., Cesaroni, R., Rioja, M.J. 2005, $A \& A$ in press

Motte, F., Andre, P., Neri, R. 1998, A\&A 336, 150

Olmi, L., Cesaroni, R., Walmsley, C.M. 1993, A\&A 276, 489

Olmi, L., Cesaroni, R., Neri, R., Walmsley, C.M. 1996, A\&A 315, 565

Pascucci, I., Apai, D., Henning, Th., Stecklum, B., Brandl, B. 2004, A\&A 426, 523

Plume, R., Jaffe, D. T., Evans, N. J., Martin-Pintado, J., Gomez-Gonzalez, J. 1997, ApJ 476, 730

Scalo, J. 1998, The Stellar Initial Mass Function, Eds. Gary Gilmore and Debbie Howell, ASP Conference Series 142, 201

Shepherd, D.S., Yu, K.C., Bally, J., Testi, L. 2000, ApJ 535, 833

Shu, F.H., Adams, F.C., Lizano, S. 1987, ARA $\mathcal{E} A$ 25, 23

Terebey, S., Shu, F.H., Cassen, P. 1984, ApJ 286, 529

Testi, L., \& Sargent, A.I. 1998, ApJ 508, L91

Walsh, A.J., Burton, M.G., Hyland, A.R., Robinson, G. 1998, MNRAS 301, 640

Watt, S. \& Mundy, L. G. 1999, ApJS 125, 143

Wood, D.O.S. \& Churchwell, E. 1998, ApJ 340, 265

Zhang, Qizhou, Ho, Paul T. P., Ohashi, Nagayoshi 1998a, ApJ 505, L151

Zhang, Q., Hunter, T.R., Sridharan, T.K. 1998, ApJ 505, L151 1998b, ApJ 505, L151 